\title{
Fine-Filtered Attributed Key Based Data Storage in
}

\section{Cloud Computing}

\author{
Lohit Krishna1)
}

\begin{abstract}
With the improvement of cloud computing, outsourcing information to cloud server draws in bunches of considerations. To ensure the security and accomplish flexibly fine-grained file access control, attribute based encryption (ABE) was proposed and utilized as a part of distributed storage system. Be that as it may, client denial is the essential issue in $\mathrm{ABE}$ plans. In this article, we give a ciphertext-policy attribute based encryption (CP-ABE) scheme with productive client denial for distributed storage system. The issue of client repudiation can be understood productively by presenting the idea of client gathering. At the point when any client leaves, the gathering chief will overhaul clients' private keys with the exception of the individuals who have been repudiated. Also, CP-ABE plot has substantial calculation cost, as it develops directly with the multifaceted nature for the get to structure. To decrease the calculation cost, we outsource high calculation load to cloud specialist co-ops without spilling record substance and mystery keys. Notbaly, our plan can withstand plot assault performed by denied clients participating with existing clients. We demonstrate the security of our plan under the detachable calculation Diffie-Hellman (DCDH) presumption. The consequence of our analysis demonstrates calculation cost for neighborhood gadgets is generally low and can be consistent. Our plan is appropriate for resource constrained devices.
\end{abstract}

Keywords :cloud computing, attribute-based encryption, outsource decryption, user revocation, collusion attack.

\section{Introduction}

Cloud computing is viewed as a prospective computing paradigm in which resource is provided as administration over the Internet. It has met the expanding needs of processing assets and capacity assets for a few undertakings because of its focal points of economy, scalability, and accessibility. As of late, a few distributed storage administrations, for example, Microsoft Azure and Google App Engine were assembled and can supply clients with scalable and dynamic storage.

Received(May 23, 2016), Review Result(1st: June 10, 2016, 2nd: July 15, 2016), Accepted(September 10, 2016)

${ }^{1}$ (Corresponding Author) Machine Intelligence Research Labs, India

email: lohitkrishna39@gmail.com 
With the expanding of delicate information outsourced tocloud, cloud storage administrations are confronting many difficulties including information security and information get to control. To take care of those issues, attribute-based encryption (ABE) plans [1-3] have been connected to distributed storage administrations. Sahai and Waters[1] initially proposed ABE conspire named fluffy personality based encryption which is gotten from identity-based encryption (IBE) [4]. As another proposed cryptographic primitive, ABE conspire has the upside of IBE plan, as well as gives the normal for "one-to-numerous" encryption. By and by, ABE chiefly incorporates two classes called ciphertext-approach $\mathrm{ABE}$ (CP-ABE) [2] and key-arrangement $\mathrm{ABE}$ (KP-ABE) [3]. In $\mathrm{CP}-\mathrm{ABE}$, ciphertexts are related with get to approaches and client's private keys are related with quality sets. A client can unscramble the ciphertext if his characteristics fulfill the get to arrangement installed in the ciphertext. It is opposite in $\mathrm{KP}-\mathrm{ABE}$. $\mathrm{CP}-\mathrm{ABE}$ is more appropriate for the outsourcing information engineering than $\mathrm{KP}-\mathrm{ABE}$ on the grounds that they get to arrangement is characterized by the information proprietors. In this article, we exhibit a proficient $\mathrm{CP}-\mathrm{ABE}$ with client revocation ability.

\section{Proposed system}

\subsection{Related Work}

In spite of the fact that $\mathrm{ABE}$ has demonstrated its benefits, client disavowal and characteristic renouncement are the essential concerns. The disavowal issue is considerably more troublesome particularly in $\mathrm{CP}-\mathrm{ABE}$ plans, in light of the fact that every property is shared by numerous clients. This implies repudiation for any trait or any single client may influence alternate clients in the framework. As of late, some work [5-9] has been proposed to take care of this issue in productive ways. Boldyreva et al. [5] gave an IBE conspire productive repudiation, which is additionally appropriate for KP-ABE. All things considered, it is uncertain whether their plan is reasonable for $\mathrm{CP}-\mathrm{ABE}$. $\mathrm{Yu}$ et al. [6] gave a trait based information imparting plan to characteristic repudiation capacity. This plan was turned out to be secure against picked plaintext assaults (CPA) in light of DBDH supposition. In any case, the length of ciphertext and client's private key are relative to the quantity of traits in the characteristic universe. In the key era, encryption and unscrambling stages, calculation includes all properties in the trait universe. Subsequently, it is costly in correspondence and calculation cost for clients. Tysowski et al. [8] gave a simple strategy to perform client repudiation operation by consolidating 
$\mathrm{CP}-\mathrm{ABE}$ with re-encryption. In their plan, every client has a place with a gathering and holds a gathering mystery key issued by the gathering. Be that as it may, their plan does not avoid arrangement assault performed by revoked clients participating with existing clients. The reason is that every client's gathering mystery key is same in a similar gathering. The qualities of the renounced clients can be utilized by the client in a similar gathering without the predetermined traits. Furthermore, we call attention to that there is a similar security hazard in the plans [7] [9].

\subsection{Existing System}

Boldyreva et al. [5] given an IBE scheme with efficient revocation, which is also suitable for $\mathrm{KP}-\mathrm{ABE}$. In any case, it is uncertain whether their plan is reasonable for CP-ABE.

$\mathrm{Yu}$ et al. [6] given a property based information offering plan to quality renouncement capacity. This plan was ended up being secure against picked plaintext assaults (CPA) in light of $\mathrm{DBDH}$ suspicion. Be that as it may, the length of figure content and client's private key are relative to the quantity of traits in the characteristic universe.

$\mathrm{Yu}$ et al. [6] planned a KP-ABE conspire with fine-grained information get to control. This plan requires that the root hub in the get to tree is an AND door and one kid isa leaf hub which is related with the fake characteristic.

In the current scheme, when a client leaves from a client gathering, the gathering supervisor just repudiates his gathering mystery key which suggests that the client's private key related with characteristics is still legitimate[10-12]. In the event that somebody in the gathering deliberately uncovered the gathering mystery key to the denied client, he can perform decoding operations through his private key[13-15]. To illuminate this assault, a solid example is given. Expect that the information is encoded under the arrangement "teacher AND cryptography" and the gathering open key. Assume that there are two clients: userland user2 whose private keys are related with the quality sets \{male, educator, cryptography\} and \{male, understudy, cryptography\} individually. On the off chance that the two are in the gathering and hold the gathering mystery key, then user1can unscramble the information however user2 can't. At the point when userlis renounced from the gathering, he can't unscramble alone on the grounds that he doesn't have the overhauled aggregate mystery key. Be that as it may, the traits of user1are not renounced and user2 has the upgraded aggregate mystery key. In this way, user1can connive with user2 to play out the decoding operation. Moreover, security model and verification were not given in their scheme[16-20]. 


\subsubsection{Disadvantage of Existing System}

It is costly in communication and computation cost for clients. Unfortunately, ABE scheme requires high calculation overhead amid performing encryption and unscrambling operations. This deformity turns out to be more serious for lightweight gadgets because of their compelled registering assets.

There is a major limitation to single-authority $\mathrm{ABE}$ as in IBE. To be specific, every client validates him to the expert, demonstrates that he has a specific property set, and afterward gets mystery key related with each of those characteristics. In this way, the specialist must be trusted to screen every one of the traits. It is unreasonable in practice and cumbersome for authority.

\subsection{Proposed System}

In this system, we concentrate on outlining a CP-ABE scheme with effective client disavowal for distributed storage system. We mean to model collusion attack performed by revoked clients coordinating with existing clients.

Furthermore, we build an effective user revocation $\mathrm{CP}-\mathrm{ABE}$ scheme through improving the existing scheme and demonstrate our plan is CPA secure under the specific model.

To solve existing security issue, we implant an endorsement into every client's private key. Along these lines, every client's gathering mystery key is unique in relation to others and bound together with his private key related with attributes.

To lessen clients' computation loads, we present two cloud specialist organizations named encryption-cloud service provider (E-CSP) and decryption-cloud service provider (D-CSP). The obligation of E-CSP is to perform outsourced encryption operation and D-CSP is to perform outsourced unscrambling operation.

In the encryption stage, the operation related with the spurious property is performed locally while the operation related with the sub-tree is outsourced to E-CSP.

\subsubsection{Advantages of Proposed System}

Reduction of the heavy computation load on clients. We outsource the majority of calculation load to E-CSP and D-CSP and leave little computation cost to local devices.

Our plan is effective for resource constrained devices such as mobile phones. Our plan can 
be utilized as a part of distributed storage system that requires the capacities of client renouncement and fine-grained access control.

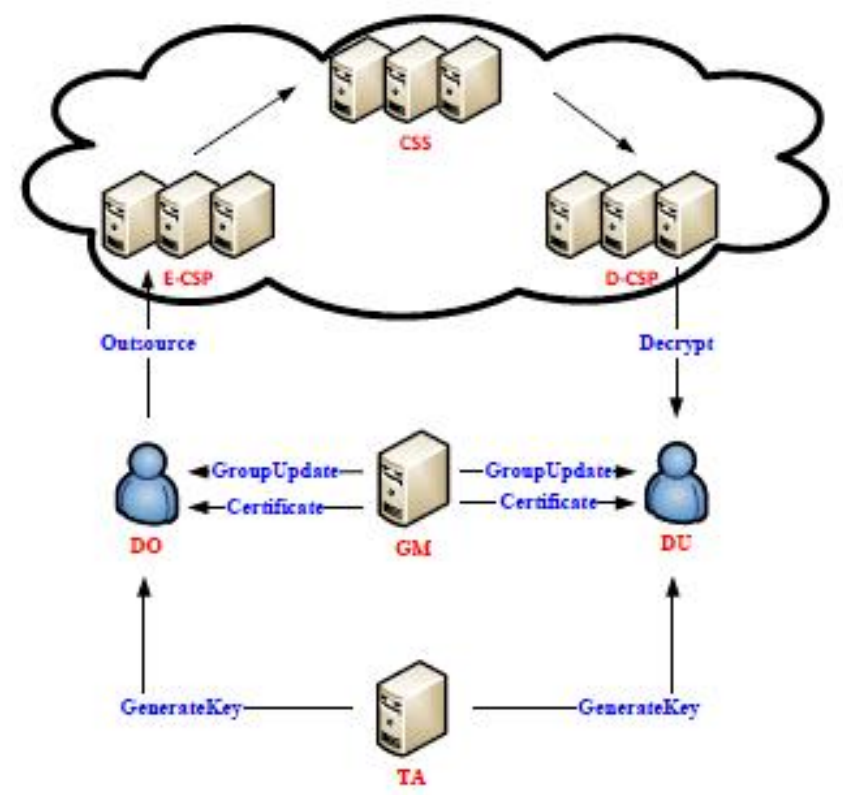

[Fig. 1] System Architecture

\section{Conclusion}

In this article, we gave a formal definition and security show for CP-ABE with client revocation. We additionally build a solid CP-ABE scheme which is CPA secure in light of $\mathrm{DCDH}$ presumption. To oppose plot assault, we install an authentication into the client's private key. So that vindictive clients and the renounced clients don't be able to create a legitimate private key through joining their private keys. Also, we outsource operations with high calculation cost to E-CSP and D-CSP to diminish the client's calculation loads. Through applying the system of outsource, computation cost for nearby devices is much lower and moderately settled. The aftereffects of our examination demonstrate that our plan is proficient for resource constrained devices.

\section{References}


[1] A. Sahai and B. Waters, Fuzzy Identity-Based Encryption, EUROCRYPT'05, LNCS, (2005), Vol.3494, pp.457-473.

[2] J.Bethencourt, A. Sahai and B. Waters, Ciphertext-Policy Attribute-Based Encryption, Proc. IEEE Symposium on Security and Privacy, (2007) May, pp.321-334, doi: 10.1109/SP.2007.11.

[3] V. Goyal, O. Pandey, A. Sahai, and B. Waters, Attribute-Based En-cryption for Fine-Grained Access Control of Encrypted Data Proc. 13th ACM Conference on Computer and Communications Security (CCS '06), (2006), pp. 89-98, doi:10.1145/1180405.1180418.

[4] D. Boneh and M. K. Franklin, Identity-Based Encryption from the Weil Pairing, SIAM Journal of Computing, (2003), Vol.32, No.3, pp.586-615.

[5] A. Boldyreva, V. Goyal, and V. Kumar, Identity-Based En-cryption with Efficient Revocation Proc.15th ACM conference on Computer and communications security (CCS' 08), (2008), pp.417-426.

[6] S. Yu, C. Wang, K. Ren, and W. Lou, Attribute Based Data Sharing with Attribute Revocation, Proc.5th ACM Symposium on Information, Computer and Communications Security (ASIACCS' 10), (2010), pp. 261-270.

[7] M. Yang, F. Liu, J. Han, and Z. Wang, An Efficient Attribute based Encryption Scheme with Revocation for Outsourced Data Sharing Control, Proc. 2011 International Conference on Instru-mentation, Measurement, Computer, Communication and Control, (2011), pp.516-520.

[8] P. K. Tysowski and M. A. Hasan, Hybrid Attribute-Based Encryption and Re-Encryption for Scalable Mobile Applications in Clouds, IEEE Transactions on Cloud Computing, (2013), pp.172-186.

[9] J. Hur and D. K. Noh, Attribute-Based Access Control with Efficient Revocation in Data Outsourcing Systems, IEEE Transactions on Parallel and Distributed Systems, (2011), pp.1214-1221.

[10] S. Yu, C. Wang, K. Ren, and W. Lou, Achieving Secure, Scalable, and Fine-Grained Data Access Control in Cloud Computing, Proc. of IEEE INFOCOM'10, (2010), pp.1-9.

[11] M. Green, S. Hohenbergerand B. Waters, Outsourcing the decryption of ABE ciphertexts, Proc. 20th USENIX Conference on Security (SEC '11), (2011), pp.34.

[12] J. Li, X. F. Chen, J.W. Li, C.F. Jia, J.F. Ma, and W. J. Lou, Fine-Grained Access Control System Based on Outsourced Attribute-Based Encryp-tion, Proc.18th European Symposium on Research in Computer Security (ESORICS' 13), LNCS8134, (2013), pp. 592-609; Berlin, Germany

[13] J. W. Li, C. F. Jia, J. Liand, and X. F. Chen, Outsourcing Encryption of At-tribute-Based Encryption with Mapreduce, Proc. 14th International Conferenceon Information and Communications Security (ICICS '12), LNCS7618, Berlin:Springer-Verlag, (2012), pp.191-201, doi:10.1007/978-3-642-34129-8_17

[14] M. Chase, Multi-authority Attribute Based Encryption, Proc. 4th Theory of Cryptography Conference (TCC '07), LNCS4392, Berlin:Springer-Verlag, (2007), pp.515-534.

[15] Z. Liu, Z. Cao, Q. Huang, D. S. Wongand, and T. H. Yuen, Fully Secure Multi-Authority Ciphertext-Policy Attribute-Based Encryption without Random Oracles, Proc.16th European Symposium on Research in Computer Security (ESORICS '11), LNCS6879, Berlin:Springer-Verlag, (2011), pp. 278-297.

[16] J. G. Han, W. Susilo, Y. Mu, and J. Yan, Privacy-Preserving Decentralized Key-Policy Attribute-Based 
Encryption, IEEE Transactions on Parallel and Distributed Systems, (2012), Vol.23, No.11, pp.2150-2162, doi: $10.1109 /$ TPDS.2012.50.

[17] H. L. Qian, J. G. Liand, and Y. C. Zhang, Privacy-Preserving Decentralized Ciphertext-Policy Attribute-Based Encryption with Fully Hidden Access Structure, Proc.15th International Conference on Information and Communications Security (ICICS '13), LNCS8233, Berlin:Springer-Verlag, (2013), pp.363-372.

[18] H. L. Qian, J. G. Li, Y. C. Zhangand, and J.G. Han, Privacy Preserving Personal Health Record Using Multi-Authority Attribute-Based Encryption with Revocation, International Journal of Information Security, doi:10.1007/s10207-014-0270-9.

[19] Z. Liu, Z. F. Cao, and D. S. Wong, Blackbox Traceable CP-ABE: How to Catch People Leaking Their Keys by Selling Decryption Devices on eBay, Proc. 2013 ACM SIGSAC Conference on Computer and Communications Security (CCS '13), (2013), pp.475-486, doi: 10.1145/2508859.2516683.

[20] Z. Liu, Z. F. Cao, and D. S. Wong, White-Box Traceable Ciphertext-Policy Attribute-Based Encryption Supporting Any Monotone Access Structures, IEEE Transactions on Information Forensics and Security, (2013), Vol.8, No.1, pp.76-88, doi: 10.1109/TIFS.2012.2223683. 
Fine-Filtered Attributed Key Based Data Storage in Cloud Computing

(This page is empty intentionally) 